\title{
Germline TYK2 mutation and cancer risk
}

James Yarmolinsky, $\mathrm{PhD}^{1,2}$, Christopher I. Amos, $\mathrm{PhD}^{3}$, Rayjean J. Hung, $\mathrm{PhD}^{4,5}$, Victor Moreno, $\mathrm{PhD}^{6-9}$, Kimberley Burrows, $\mathrm{PhD}^{1,2}$, Karl Smith-Byrne, DPhil ${ }^{10}$, Joshua R. Atkins, $\mathrm{PhD}^{11}$, Paul Brennan, $\mathrm{PhD}^{11}$, Colon Cancer Family Registry (CCFR), Colorectal Cancer Transdisciplinary study (CORECT), Genetics and Epidemiology of Colorectal Cancer Consortium (GECCO), Prostate Cancer Association Group to Investigate Cancer Associated Alterations in the Genome (PRACTICAL) consortium, James D. McKay, $\mathrm{PhD}^{11}$, Richard M. Martin, BMBS, PhD ${ }^{1,2,12}$, George Davey Smith, $\mathrm{FRS}, \mathrm{MD}, \mathrm{DSc}^{1,2}$

${ }^{1}$ Medical Research Council Integrative Epidemiology Unit at the University of Bristol, Bristol, United Kingdom

${ }^{2}$ Population Health Sciences, Bristol Medical School, University of Bristol, Bristol, United Kingdom

${ }^{3}$ Baylor College of Medicine, Houston, USA

${ }^{4}$ Lunenfeld-Tanenbaum Research Institute, Sinai Health, Toronto, Canada

${ }^{5}$ University of Toronto, Toronto, Canada

${ }^{6}$ Biomarkers and Susceptibility Unit, Oncology Data Analytics Program, Catalan Institute of Oncology (ICO), L'Hospitalet de Llobregat, Barcelona, Spain

${ }^{7}$ Colorectal Cancer Group, ONCOBELL Program, Bellvitge Biomedical Research Institute (IDIBELL), L'Hospitalet de Llobregat, Barcelona, Spain

${ }^{8}$ Consortium for Biomedical Research in Epidemiology and Public Health (CIBERESP), Madrid, Spain

${ }^{9}$ Department of Clinical Sciences, Faculty of Medicine, University of Barcelona, Barcelona, Spain 
${ }^{10}$ Cancer Epidemiology Unit, Oxford Population Health, University of Oxford

${ }^{11}$ Genomic Epidemiology Branch, International Agency for Research on Cancer (IARC/WHO), Lyon,

France

${ }^{12}$ University Hospitals Bristol, NHS Foundation Trust, National Institute for Health Research Bristol

Biomedical Research Centre, University of Bristol, Bristol, UK

Members of the Colon Cancer Family Registry (CCFR), Colorectal Cancer Transdisciplinary study

(CORECT), Genetics and Epidemiology of Colorectal Cancer Consortium (GECCO), and Prostate

Cancer Association Group to Investigate Cancer Associated Alterations in the Genome (PRACTICAL)

consortium are provided in Supplementary Material. Further information on the PRACTICAL

consortium can be found at http://practical.icr.ac.uk/.

Word count: 922

\section{Corresponding author:}

James Yarmolinsky, PhD

MRC Integrative Epidemiology Unit

Population Health Sciences

Bristol Medical School

University of Bristol

Bristol, UK

james.yarmolinsky@bristol.ac.uk 


\begin{abstract}
Deucravacitinib, a novel, selective inhibitor of TYK2 is currently under review at the FDA and EMA for treatment of moderate-to-severe plaque psoriasis. It is unclear whether recent safety concerns (i.e. elevated rates of lung cancer and lymphoma) related to similar medications (i.e. other JAK inhibitors) are shared with this novel TYK2 inhibitor. We used a partial loss-of-function variant in TYK2 (rs34536443), previously shown to protect against psoriasis and other autoimmune diseases, to evaluate the potential effect of therapeutic TYK2 inhibition on risk of lung cancer and non-Hodgkin lymphoma. Summary genetic association data on lung cancer risk were obtained from a GWAS metaanalysis of 29,266 cases and 56,450 controls in the Integrative Analysis of Lung Cancer Risk and Etiology (INTEGRAL) consortium. Summary genetic association data on non-Hodgkin lymphoma risk were obtained from a GWAS meta-analysis of 8,489 cases and 374,506 controls in the UK Biobank and InterLymph consortium. In the primary analysis, each copy of the minor allele of rs34536443, representing partial TYK2 inhibition, was associated with an increased risk of lung cancer (OR 1.15, 95\% CI 1.07-1.24, P=1.72 x 10 $0^{-4}$ ) and non-Hodgkin lymphoma (OR 1.18, 95\% CI $\left.1.05-1.33, P=5.25 \times 10^{-3}\right)$. In secondary analyses, there was weak evidence of an association of rs34536443 with advanced prostate cancer risk (OR 1.08, 95\% CI 1.00-1.17, $P=0.04$ ), but little evidence of association with three other common adult cancers examined. Our analyses using an established partial loss-of-function mutation to mimic TYK2 inhibition provide genetic evidence that therapeutic TYK2 inhibition may increase risk of lung cancer and non-Hodgkin lymphoma. These findings, consistent with recent reports from post-marketing trials of similar JAK inhibitors, could have important implications for future safety assessment of Deucravacitinib and other TYK2 inhibitors in development.
\end{abstract}




\section{Background}

On September 1, 2021 the U.S. Food and Drug Administration (FDA) announced that three Janus kinase (JAK) inhibitors approved to treat chronic inflammatory conditions would require safety warnings over increased rates of serious heart-related events, lung cancer, and lymphoma ${ }^{1}$. Recently, oral Deucravacitinib, a selective inhibitor of Tyrosine kinase 2 (TYK2, a member of the JAK family), was shown to lead to larger improvements in symptom severity for patients with moderate-to-severe plaque psoriasis than oral Apremilast, the current standard of care ${ }^{2,3}$. Deucravacitinib, therefore, has the potential to become an important treatment option for patients with psoriasis requiring systemic treatment and is currently under review for approval at the FDA and European Medicines Agency ${ }^{2}$. It is unclear, however, whether the elevated cancer risk associated with some JAK inhibitors is shared with this novel TYK2 inhibitor.

In the absence of long-term clinical trial data, naturally occurring genetic variation can be leveraged to validate therapeutic targets and predict their adverse effects ${ }^{4}$. Specifically, germline mutations causing partial or complete loss-of-function (LOF) of genes encoding drug targets can be employed to mimic pharmacological inhibition of these targets and have been used to correctly predict adverse effects of new medications ${ }^{5}$.

Here, we used an established partial LOF mutation in TYK2 (rs34536443), previously shown to protect against psoriasis and other autoimmune diseases ${ }^{6,7}$, to evaluate the potential effect of therapeutic TYK2 inhibition on risk of lung cancer and non-Hodgkin lymphoma.

\section{Methods}

Minor allele homozygosity of rs34536443 causes near-complete ( $80 \%)$ loss of TYK2 function, while heterozygotes have a more modest reduction in function $(<40 \%)$, suggesting nonadditive effects of this variant ${ }^{6}$. To validate this variant as a surrogate for therapeutic TYK2 inhibition, we evaluated the effect of each copy of the minor allele of rs34536443, representing partial TYK2 inhibition, on risk of psoriasis, inflammatory bowel disease, Crohn's disease, and multiple sclerosis. 
These analyses were performed using summary genetic association data on up to 78,334 cases and 150,030 controls from genome-wide association studies (GWAS) of these autoimmune diseases ${ }^{8-11}$.

To evaluate the effect of this variant on risk of overall and histological subtype-specific lung cancer we obtained summary genetic association data on up to 29,266 cases and 56,450 controls from a GWAS meta-analysis of the Integrative Analysis of Lung Cancer Risk and Etiology (INTEGRAL) ${ }^{12}$. Summary genetic association data for non-Hodgkin lymphoma (NHL) were generated by metaanalysing genome-wide association (GWAS) data for rs34536443 from the UK Biobank and InterLymph consortium in METAL ${ }^{13}$.

In secondary analyses, we explored whether rs34536443 increased risk of three other common adult cancers (breast, colorectal, prostate), which, along with lung cancer, account for approximately half of all new U.S. cancer cases ${ }^{14}$. Summary genetic association data on overall and histological subtype-specific breast, colorectal, and prostate cancer on up to 260,346 cases and 234,774 controls were obtained from analyses of the Breast Cancer Association Consortium (BCAC), Genetics and Epidemiology of Colorectal Cancer Consortium (GECCO), ColoRectal Cancer Transdisciplinary Study (CORECT), Colon Cancer Family Registry (CCFR), and the Prostate Cancer Association Group to Investigate Cancer Associated Alterations in the Genome (PRACTICAL) consortium $^{15-17}$.

All analyses were restricted to participants of European ancestry. Further information on statistical analysis, imputation, and quality control measures for these studies is provided in Supplementary Material and in the original publications. All studies contributing data to these analyses had the relevant institutional review board approval from each country, in accordance with the Declaration of Helsinki, and all participants provided informed consent.

\section{Results}


To validate rs34536443 as a surrogate for therapeutic TYK2 inhibition, we confirmed that each copy of the minor allele, representing partial TYK2 inhibition, was associated with lower risk of psoriasis (OR 0.48, 95\% CI 0.39-0.58, $P=9.01 \times 10^{-14}$ ) and other autoimmune diseases (Table 1).

In analyses of 29,266 lung cancer cases and 56,540 controls, each copy of the minor allele of rs34536443 was associated with an increased risk of lung cancer (OR 1.15, 95\% CI 1.07-1.24, $P=$ $1.72 \times 10^{-4}$ ). This association was stronger for current and former smokers (OR 1.15, 95\% CI 1.09$\left.1.23, P=2.29 \times 10^{-6}\right)$ compared to never smokers (OR 1.09, 95\% CI 0.91-1.32, $P=0.34$ ). The magnitude of effect was similar across histology types. In analyses of 8,489 cases and 374,506 controls, each copy of the minor allele of rs34536443 was associated with an increased risk of nonHodgkin lymphoma (OR 1.18, 95\% CI 1.05-1.33, $P=5.25 \times 10^{-3}$ ). In secondary analyses, rs34536443 was weakly associated with risk of advanced prostate cancer, but not associated with other cancers examined.

\section{Discussion}

The efficacy of Deucravactinib in treating plaque psoriasis is attributed to the selective inhibition of TYK2, a downstream mediator of pro-inflammatory signalling pathways critical to psoriasis $^{3}$. Using an established partial loss-of-function variant to mimic therapeutic TYK2 inhibition, we show that potential protection from autoimmunity mediated by TYK2 inhibition may be counteracted by an increased risk of lung cancer and non-Hodgkin lymphoma. This finding, including the restriction of an increased lung cancer risk to current and former smokers, is consistent with recent reports of higher rates of these two cancers in users of the JAK inhibitor Tofacitinib in the ORAL

Surveillance safety trial ${ }^{1}$. Importantly, the per-allele estimates presented in this analysis may underestimate the effect of therapeutic TYK2 inhibition on cancer given larger TYK2 reductions achieved by Deucravacitinib at doses shown to confer therapeutic benefit (50-80\% TYK2 inhibition) compared with the $<40 \%$ expected per copy of the rs 34536443 minor allele ${ }^{18}$. These findings, suggesting potential adverse target-mediated effects of TYK2 inhibition on lung cancer and non- 
medRxiv preprint doi: https://doi.org/10.1101/2022.01.26.22269888; this version posted January 29, 2022. The copyright holder for this preprint (which was not certified by peer review) is the author/funder, who has granted medRxiv a license to display the preprint in perpetuity.

It is made available under a CC-BY-NC-ND 4.0 International license.

Hodgkin lymphoma, could have important implications for future safety assessment of

Deucravacitinib and other TYK2 inhibitors in development. 
Table 1. Effect of the minor allele of TYK2 variant rs34536443 on risk of autoimmune conditions, lung cancer and non-Hodgkin lymphoma, and other common adult cancers

\begin{tabular}{|c|c|c|c|}
\hline Outcome & $\mathbf{N}$ (cases, controls) & OR $(95 \%$ CI $)$ & $P$-value \\
\hline \multicolumn{4}{|l|}{ Autoimmune conditions } \\
\hline Psoriasis & 10,$588 ; 22,806$ & $0.48(0.39-0.58)$ & $9.01 \times 10^{-14}$ \\
\hline Crohn's disease & 5,$956 ; 14,927$ & $0.65(0.55-0.76)$ & $3.29 \times 10^{-7}$ \\
\hline Multiple sclerosis & 47,$429 ; 68,374$ & $0.77(0.69-0.85)$ & $2.87 \times 10^{-7}$ \\
\hline Rheumatoid arthritis & 14,$361 ; 43,923$ & $0.68(0.63-0.75)$ & $4.60 \times 10^{-16}$ \\
\hline \multicolumn{4}{|l|}{ Primary cancer outcomes } \\
\hline Lung cancer & 29,$266 ; 56,450$ & $1.15(1.07-1.24)$ & $1.72 \times 10^{-4}$ \\
\hline Current or former smokers & 23,$223 ; 16,964$ & $1.15(1.09-1.23)$ & $2.29 \times 10^{-6}$ \\
\hline Never smokers & 2,$355 ; 7,504$ & $1.09(0.91-1.32)$ & 0.34 \\
\hline Lung adenocarcinoma & 11,$273 ; 55,483$ & $1.17(1.08-1.27)$ & $1.37 \times 10^{-4}$ \\
\hline Squamous cell carcinoma & 7,$426 ; 55,627$ & $1.19(1.08-1.31)$ & $2.98 \times 10^{-4}$ \\
\hline Small cell lung cancer & 2,$664 ; 21,444$ & $1.16(1.00-1.34)$ & 0.05 \\
\hline Non-Hodgkin lymphoma & 8,$489 ; 374,506$ & $1.18(1.05-1.33)$ & $5.25 \times 10^{-3}$ \\
\hline \multicolumn{4}{|l|}{ Secondary cancer outcomes } \\
\hline Breast cancer & 122,$977 ; 105,974$ & $0.99(0.96-1.03)$ & 0.69 \\
\hline ER+ Breast cancer & 69,$501 ; 105,974$ & $1.00(0.96-1.04)$ & 0.95 \\
\hline ER- Breast cancer & 21,$468 ; 105,974$ & $1.00(0.96-1.04)$ & 0.96 \\
\hline Colorectal cancer & 58,$221 ; 67,694$ & $1.03(0.99-1.08)$ & 0.18 \\
\hline Colon cancer & 32,$002 ; 64,159$ & $1.02(0.97-1.08)$ & 0.39 \\
\hline Rectal cancer & 16,$212 ; 64,159$ & $1.05(0.98-1.13)$ & 0.15 \\
\hline Prostate cancer & 79,$148 ; 61,106$ & $1.04(1.00-1.09)$ & 0.07 \\
\hline
\end{tabular}


medRxiv preprint doi: https://doi.org/10.1101/2022.01.26.22269888; this version posted January 29, 2022. The copyright holder for this preprint (which was not certified by peer review) is the author/funder, who has granted medRxiv a license to display the preprint in perpetuity. It is made available under a CC-BY-NC-ND 4.0 International license .

\begin{tabular}{|l|l|l|l|}
\hline Advanced prostate cancer & 15,$167 ; 58,308$ & $1.08(1.00-1.17)$ & 0.04 \\
\hline
\end{tabular}

OR represents the exponential change in odds of cancer per each copy of the minor allele of rs34536443.

Advanced prostate cancer defined as Gleason score $\geq 8$, prostate-specific antigen $>100 \mathrm{ng} / \mathrm{mL}$, metastatic disease (M1), or death from prostate cancer. 


\section{Citations}

1. $\quad$ U.S. Food and Drug Administration. FDA requires warnings about increased risk of serious heart-related events, cancer, blood clots, and death for JAK inhibitors that treat certain chronic inflammatory conditions. 2021.

2. $\quad$ Business Wire. Bristol Myers Squibb's Applications for Deucravacitinib for the Treatment of Moderate to Severe Plaque Psoriasis Accepted by U.S. Food and Drug Administration and Validated by European Medicines Agency. Retrieved on 8 December 2021 from: https://www.businesswire.com/news/home/20211129005192/en/Bristol-MyersSquibb\%E2\%80\%99s-Applications-for-Deucravacitinib-for-the-Treatment-of-Moderate-toSevere-Plaque-Psoriasis-Accepted-by-U.S.-Food-and-Drug-Administration-and-Validatedby-European-Medicines-Agency. Accessed.

3. Armstrong A, Gooderham M, Warren RB, et al. POS1042 EFFICACY AND SAFETY OF DEUCRAVACITINIB, AN ORAL, SELECTIVE TYROSINE KINASE 2 (TYK2) INHIBITOR, COMPARED WITH PLACEBO AND APREMILAST IN MODERATE TO SEVERE PLAQUE PSORIASIS: RESULTS FROM THE PHASE 3 POETYK PSO-1 STUDY. Annals of the Rheumatic Diseases. 2021;80(Suppl 1):795-796.

4. Holmes MV, Richardson TG, Ference BA, Davies NM, Davey Smith G. Integrating genomics with biomarkers and therapeutic targets to invigorate cardiovascular drug development. Nat Rev Cardiol. 2021;18(6):435-453.

5. Minikel EV, Karczewski KJ, Martin HC, et al. Evaluating drug targets through human lossof-function genetic variation. Nature. 2020;581(7809):459-464.

6. Dendrou CA, Cortes A, Shipman L, et al. Resolving TYK2 locus genotype-to-phenotype differences in autoimmunity. Sci Transl Med. 2016;8(363):363ra149.

7. Diogo D, Bastarache L, Liao KP, et al. TYK2 protein-coding variants protect against rheumatoid arthritis and autoimmunity, with no evidence of major pleiotropic effects on nonautoimmune complex traits. PLoS One. 2015;10(4):e0122271.

8. International Multiple Sclerosis Genetics Consortium. Multiple sclerosis genomic map implicates peripheral immune cells and microglia in susceptibility. Science. 2019;365(6460).

9. Liu JZ, van Sommeren S, Huang H, et al. Association analyses identify 38 susceptibility loci for inflammatory bowel disease and highlight shared genetic risk across populations. Nat Genet. 2015;47(9):979-986.

10. Okada Y, Wu D, Trynka G, et al. Genetics of rheumatoid arthritis contributes to biology and drug discovery. Nature. 2014;506(7488):376-381.

11. Tsoi LC, Spain SL, Knight J, et al. Identification of 15 new psoriasis susceptibility loci highlights the role of innate immunity. Nat Genet. 2012;44(12):1341-1348.

12. McKay JD, Hung RJ, Han Y, et al. Large-scale association analysis identifies new lung cancer susceptibility loci and heterogeneity in genetic susceptibility across histological subtypes. Nat Genet. 2017;49(7):1126-1132.

13. Morton LM, Sampson JN, Cerhan JR, et al. Rationale and Design of the International Lymphoma Epidemiology Consortium (InterLymph) Non-Hodgkin Lymphoma Subtypes Project. J Natl Cancer Inst Monogr. 2014;2014(48):1-14.

14. Siegel RL, Miller KD, Jemal A. Cancer statistics, 2020. CA Cancer J Clin. 2020;70(1):7-30.

15. Huyghe JR, Bien SA, Harrison TA, et al. Discovery of common and rare genetic risk variants for colorectal cancer. Nat Genet. 2019;51(1):76-87.

16. Michailidou K, Lindström S, Dennis J, et al. Association analysis identifies 65 new breast cancer risk loci. Nature. 2017;551(7678):92-94.

17. Schumacher FR, Al Olama AA, Berndt SI, et al. Association analyses of more than 140,000 men identify 63 new prostate cancer susceptibility loci. Nat Genet. 2018;50(7):928-936.

18. Papp K, Gordon K, Thaçi D, et al. Phase 2 Trial of Selective Tyrosine Kinase 2 Inhibition in Psoriasis. N Engl J Med. 2018;379(14):1313-1321. 


\section{Funding}

JY is supported by a Cancer Research UK Population Research Postdoctoral Fellowship (C68933/A28534). JY, RMM, and GDS are supported by a Cancer Research UK (C18281/A29019) programme grant (the Integrative Cancer Epidemiology Programme). JY, KB, RMM, and GDS are part of the Medical Research Council Integrative Epidemiology Unit at the University of Bristol which is supported by the Medical Research Council MC_UU_00011/1, MC_UU_00011/5) and the University of Bristol. RMM is also supported by the NIHR Bristol Biomedical Research Centre which is funded by the NIHR and is a partnership between University Hospitals Bristol NHS Foundation Trust and the University of Bristol. The US National Cancer Institute supports JM and PB (UO1CA203654) and JM (UO1CA257679). Department of Health and Social Care disclaimer: The views expressed are those of the author(s) and not necessarily those of the NHS, the NIHR, the International Agency for Research on Cancer or the Department of Health and Social Care.

\section{Acknowledgements}

The authors would like to thank the participants of the individual studies contributing to the BCAC, GECCO, CORECT, CCFR, IIBDGC, IMSGC, INTEGRAL-ILCCO, InterLymph, PRACTICAL, and UK Biobank studies. The authors would also like to acknowledge the investigators of these consortia and studies for generating the data used for this analysis. The authors would like to acknowledge the following investigators of the OncoArray and GAME-ON1KG INTEGRAL-ILCCO analyses: Maria Teresa Landi, Victoria Stevens, Ying Wang, Demetrios Albanes, Neil Caporaso, Paul Brennan, Christopher I Amos, Sanjay Shete, Rayjean J Hung, Heike Bickeböller, Angela Risch, Richard Houlston, Stephen Lam, Adonina Tardon, Chu Chen, Stig E Bojesen, Mattias Johansson, H-Erich Wichmann, David Christiani, Gadi Rennert, Susanne Arnold, John K. Field, Loic Le Marchand, Olle Melander, Hans Brunnström, Geoffrey Liu, Angeline Andrew, Lambertus A Kiemeney, Hongbing Shen, Shan Zienolddiny, Kjell Grankvist, Mikael Johansson, M Dawn Teare, Yun-Chul Hong, Jian- 
medRxiv preprint doi: https://doi.org/10.1101/2022.01.26.22269888; this version posted January 29, 2022. The copyright holder for this preprint (which was not certified by peer review) is the author/funder, who has granted medRxiv a license to display the preprint in perpetuity. It is made available under a CC-BY-NC-ND 4.0 International license.

Min Yuan, Philip Lazarus, Matthew B Schabath, Melinda C Aldrich. Non-Hodgkin lymphoma data (phs000801) was accessed using dbGaP accession number 15258. 